\title{
Constraints on the multi-TeV particle population in the Coma galaxy cluster with HESS observations
}

\author{
F. Aharonian ${ }^{1,2}$, A. G. Akhperjanian ${ }^{3}$, G. Anton ${ }^{4}$, U. Barres de Almeida ${ }^{5, \star}$, A. R. Bazer-Bachi ${ }^{6}$, Y. Becherini ${ }^{7}$,
} B. Behera ${ }^{8}$, K. Bernlöhr ${ }^{1,9}$, C. Boisson ${ }^{10}$, A. Bochow ${ }^{1}$, V. Borrel ${ }^{6}$, E. Brion ${ }^{11}$, J. Brucker ${ }^{4}$, P. Brun ${ }^{11}$, R. Bühler ${ }^{1}$, T. Bulik ${ }^{12}$, I. Büsching ${ }^{13}$, T. Boutelier ${ }^{14}$, P. M. Chadwick ${ }^{5}$, A. Charbonnier ${ }^{15}$, R. C. G. Chaves ${ }^{1}$, A. Cheesebrough ${ }^{5}$, L.-M. Chounet ${ }^{16}$, A. C. Clapson $^{1}$, G. Coignet ${ }^{17}$, M. Dalton ${ }^{9}$, M. K. Daniel ${ }^{5}$, I. D. Davids ${ }^{18,13}$, B. Degrange ${ }^{16}$, C. Deil ${ }^{1}$, H. J. Dickinson ${ }^{5}$, A. Djannati-Ataï ${ }^{7}$, W. Domainko ${ }^{1}$, L. O'C. Drury ${ }^{2}$, F. Dubois ${ }^{17}$, G. Dubus ${ }^{14}$, J. Dyks ${ }^{12}$, M. Dyrda ${ }^{19}$, K. Egberts ${ }^{1}$, D. Emmanoulopoulos ${ }^{8}$, P. Espigat ${ }^{7}$, C. Farnier ${ }^{20}$, F. Feinstein ${ }^{20}$, A. Fiasson ${ }^{20}$, A. Förster ${ }^{1}$, G. Fontaine ${ }^{16}$, M. Füßling ${ }^{9}$, S. Gabici ${ }^{2}$, Y. A. Gallant ${ }^{20}$, L. Gérard ${ }^{7}$, B. Giebels ${ }^{16}$, J. F. Glicenstein ${ }^{11}$, B. Glück ${ }^{4}$, P. Goret ${ }^{11}$, D. Göhring ${ }^{4}$, D. Hauser ${ }^{8}$, M. Hauser ${ }^{8}$, S. Heinz ${ }^{4}$, G. Heinzelmannn ${ }^{21}$, G. Henri' ${ }^{14}$, G. Hermann ${ }^{1}$, J. A. Hinton ${ }^{22}$, A. Hoffmann ${ }^{23}$, W. Hofmann ${ }^{1}$, M. Holleran ${ }^{13}$, S. Hoppe ${ }^{1}$, D. Horns ${ }^{21}$, S. Inoue ${ }^{24}$, A. Jacholkowska ${ }^{15}$, O. C. de Jager ${ }^{13}$, C. Jahn ${ }^{4}$, I. Jung ${ }^{4}$, K. Katarzyński ${ }^{25}$, U. Katz ${ }^{4}$, S. Kaufmann ${ }^{8}$, E. Kendziorra ${ }^{23}$, M. Kerschhaggl ${ }^{9}$, D. Khangulyan ${ }^{1}$, B. Khélifi ${ }^{16}$, D. Keogh ${ }^{5}$, W. Kluźniak ${ }^{12}$, T. Kneiske ${ }^{21}$, Nu. Komin ${ }^{11}$, K. Kosack ${ }^{1}$, G. Lamanna ${ }^{17}$, J.-P. Lenain ${ }^{10}$, T. Lohse ${ }^{9}$, V. Marandon ${ }^{7}$, J. M. Martin ${ }^{10}$, O. Martineau-Huynh ${ }^{15}$, A. Marcowith ${ }^{20}$, D. Maurin ${ }^{15}$, T. J. L. McComb ${ }^{5}$, M. C. Medina ${ }^{10}$, R. Moderski ${ }^{12}$, E. Moulin ${ }^{11}$, M. Naumann-Godo ${ }^{16}$, M. de Naurois ${ }^{15}$, D. Nedbal ${ }^{26}$, D. Nekrassov ${ }^{1}$, J. Niemiec ${ }^{19}$, S. J. Nolan ${ }^{5}$, S. Ohm ${ }^{1}$, J.-F. Olive ${ }^{6}$, E. de Oña Wilhelmi ${ }^{7,27}$, K. J. Orford ${ }^{5}$, M. Ostrowski ${ }^{28}$, M. Panter $^{1}$, M. Paz Arribas ${ }^{9}$, G. Pedaletti ${ }^{8}$, G. Pelletier ${ }^{14}$, P.-O. Petrucci ${ }^{14}$, S. Pita ${ }^{7}$, G. Pühlhofer ${ }^{8}$, M. Punch $^{7}$, A. Quirrenbach ${ }^{8}$, B. C. Raubenheimer ${ }^{13}$, M. Raue ${ }^{1,27}$, S. M. Rayner ${ }^{5}$, M. Renaud ${ }^{7,1}$, O. Reimer ${ }^{29}$, F. Rieger ${ }^{1,27}$, J. Ripken ${ }^{21}$, L. Rob ${ }^{26}$, S. Rosier-Lees ${ }^{17}$, G. Rowell ${ }^{30}$, B. Rudak ${ }^{12}$, C. B. Rulten ${ }^{5}$, J. Ruppel ${ }^{31}$, V. Sahakian ${ }^{3}$, A. Santangelo ${ }^{23}$,

R. Schlickeiser ${ }^{31}$, F. M. Schöck ${ }^{4}$, R. Schröder ${ }^{31}$, U. Schwanke ${ }^{9}$, S. Schwarzburg ${ }^{23}$, S. Schwemmer ${ }^{8}$, A. Shalchi ${ }^{31}$, M. Sikora ${ }^{12}$, J. L. Skilton ${ }^{22}$, H. Sol ${ }^{10}$, D. Spanglfoer ${ }^{5}$, Ł. Stawarz ${ }^{28}$, R. Steenkamp ${ }^{18}$, C. Stegmann ${ }^{4}$, G. Superina ${ }^{16}$, A. Szostek ${ }^{28,14}$, P. H. Tam ${ }^{8}$, J.-P. Tavernet ${ }^{15}$, R. Terrier ${ }^{7}$, O. Tibolla ${ }^{1,8}$, M. Tluczykont ${ }^{21}$, C. van Eldik ${ }^{1}$, G. Vasileiadis ${ }^{20}$, C. Venter $^{13}$, L. Venter ${ }^{10}$, J. P. Vialle ${ }^{17}$, P. Vincent ${ }^{15}$, M. Vivier ${ }^{11}$, H. J. Völk ${ }^{1}$, F. Volpe ${ }^{1,16,27}$, S. J. Wagner ${ }^{8}$, M. Ward ${ }^{5}$, A. A. Zdziarski ${ }^{12}$, and A. Zech ${ }^{10}$

(Affiliations can be found after the references)

Received 17 March 2009 / Accepted 19 May 2009

\section{ABSTRACT}

\begin{abstract}
Aims. Galaxy clusters are key targets in the search for ultra high energy particle accelerators. The Coma cluster represents one of the best candidates for such a search owing to its high mass, proximity, and the established non-thermal radio emission centred on the cluster core.

Methods. The HESS (High Energy Stereoscopic System) telescopes observed Coma for $\sim 8 \mathrm{~h}$ in a search for $\gamma$-ray emission at energies $>1 \mathrm{TeV}$. The large $3.5^{\circ} \mathrm{FWHM}$ field of view of HESS is ideal for viewing a range of targets at various sizes including the Coma cluster core, the radio-relic $(1253+275)$ and merger/infall (NGC 4839) regions to the southwest, and features greater than $1^{\circ}$ away.

Results. No evidence for point-like nor extended $\mathrm{TeV} \gamma$-ray emission was found and upper limits to the $\mathrm{TeV}$ flux $F(E)$ for $E>1,>5$, and $>10 \mathrm{TeV}$ were set for the Coma core and other regions. Converting these limits to an energy flux $E^{2} F(E)$ the lowest or most constraining is the $E>5 \mathrm{TeV}$

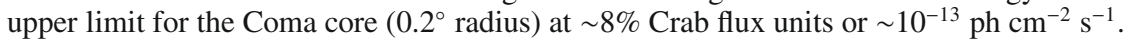

Conclusions. The upper limits for the Coma core were compared with a prediction for the $\gamma$-ray emission from proton-proton interactions, the level of which ultimately scales with the mass of the Coma cluster. A direct constraint using our most stringent limit for $E>5 \mathrm{TeV}$, on the total energy content in non-thermal protons with injection energy spectrum $\propto E^{-2.1}$ and spatial distribution following the thermal gas in the cluster, is found to be $\sim 0.2$ times the thermal energy, or $\sim 10^{62} \mathrm{erg}$. The $E>5 \mathrm{TeV} \gamma$-ray threshold in this case corresponds to cosmic-ray proton energies $\gtrsim 50 \mathrm{TeV}$. Our upper limits rule out the most optimistic theoretical models for gamma ray emission from clusters and complement radio observations which constrain the cosmic ray content in clusters at significantly lower proton energies, subject to assumptions on the magnetic field strength.
\end{abstract}

Key words. gamma rays: observations - galaxies: clusters: individual: Coma (ACO 1656)

\section{Introduction}

Clusters of galaxies represent the largest gravitationally bound objects in the Universe and are thought to be ideal sites for the acceleration of particles. The very long confinement time

^ Supported by CAPES Foundation, Ministry of Education of Brazil. (of order the Hubble time) of the accelerated particles (see e.g. Völk et al. 1996; Berezinsky et al. 1997) would allow interactions of the particles with ambient matter and radiation fields to produce non-thermal emission from radio to $\mathrm{TeV} \gamma$-ray energies. Particles are thought to be accelerated at large-scale shocks associated with accretion and merger processes (see e.g. Colafrancesco et al. 1998; Ryu et al. 2003), in supernova 
remnants and galactic-scale winds (Völk et al. 1996), turbulent re-acceleration (Brunetti \& Blasi 2005) and dark matter annihilation (e.g. Colafrancesco et al. 2006). In addition, particles may be re-distributed/injected throughout the cluster volume via AGN cluster members (Enßlin et al. 1997; Aharonian 2002; Hinton et al. 2007), The non-thermal radio emission observed in recent years from several galaxy clusters (Giovannini et al. 1993; Feretti et al. 2004) represents clear evidence for relativistic particle populations in such objects. Further evidence is provided by possible non-thermal X-rays observed from a few clusters (Rephaeli \& Gruber 2002; Fusco-Femiano et al. 2004; Eckert et al. 2007).

Gamma-ray emission in galaxy clusters may come from several processes (see review by Blasi et al. 2007). The collision of relativistic cosmic-ray (CR) protons with thermal nuclei comprising the intra-cluster medium (ICM) may lead to $\gamma$-ray emission via the decay of neutral pions (Dennison 1980). In this context the fraction $\eta$ of thermal energy in the cluster volume in the form of relativistic non-thermal particles is an important parameter that can determine the level of $\gamma$-ray emission expected. Since the thermal energy content is a function of the cluster mass, the most massive and nearby clusters present the best opportunity to probe for such $\gamma$-ray emission. Ultra-relativistic electrons can also up-scatter target photons such as the cosmic microwave background (CMB), infrared, starlight, and other soft photon fields) to TeV $\gamma$-ray energies (Atoyan \& Völk 2000; Gabici et al. 2003a, 2004). Given that galaxy clusters may accelerate particles to ultra high energies (UHE) $>10^{18} \mathrm{eV}$ (e.g. Hillas 1984; Kang et al. 1996), $\gamma$-ray production from inverseCompton scattering by secondary electrons generated when a UHE proton interacts with a CMB photon in the Bethe-Heitler process $\left(\mathrm{p} \gamma \rightarrow \mathrm{e}^{+} \mathrm{e}^{-}+\mathrm{p}^{\prime}\right.$ ) may also result (Inoue et al. 2005; Kelner \& Aharonian 2008). Dark matter annihilation has also been considered as a $\gamma$-ray production channel (e.g. neutralino annihilation by Colafrancesco et al. 2006).

Earlier observations in the $\mathrm{MeV}$ to $\mathrm{GeV} \gamma$-ray band with EGRET only found upper limits only for several clusters (Reimer et al. 2003) including the Coma cluster. At TeV energies, upper limits (Perkins et al. 2006) have been reported for the Perseus and Abell 2029 clusters using the single-dish Whipple telescope. The most recent $\mathrm{TeV}$ observations with stereoscopic instruments such as HESS (Abell 496 and Abell 85 - Aharonian et al. 2009), and with VERITAS (Coma - Perkins et al. 2008), revealed also upper limits. This work focuses on HESS observations of the Coma cluster.

Coma (ACO 1656) is one of the nearest $(z=0.023)$ and best-studied galaxy clusters. Extended (several arcminutes in scale) hard X-ray emission (with so far weak evidence for a non-thermal component) has been observed (Rephaeli \& Gruber 2002; Fusco-Feminano et al. 2004; Rossetti et al. 2004; Lutovinov et al. 2008; Ajello et al. 2009), as well as a prominent non-thermal radio halo (Giovannini et al. 1993; Thierbach et al. 2003). The latter is clear evidence for particle acceleration. Being one of the most massive $\left(M \sim 10^{15} M_{\odot}\right)$ and nearby clusters, with detailed multiwavelength observations ranging from low frequency radio wavelength to $\gamma$-rays, the Coma cluster has always been considered as the prototypical cluster also for very high energy $\gamma$-ray studies. The Coma cluster is located in the northern hemisphere and is visible by HESS at moderately high zenith angles (average value $\sim 50^{\circ}$ ), which leads to a relatively high energy threshold (defined as the peak detection rate for an $E^{-2.1}$ power-law spectrum of $\gamma$-rays) of $\gtrsim 1 \mathrm{TeV}$. Since the $\gamma$-ray spectrum from clusters is expected to be hard and extend beyond $10 \mathrm{TeV}$ (basically limited only by the absorption of $\gamma$-ray photons in the cosmic infrared background. At the Coma cluster distance, an optical depth of unity is reached for energies $E \sim 10$ to $20 \mathrm{TeV}$ ), the energy threshold does not constitute a serious problem for our investigation.

\section{HESS observations and analysis}

Operating in the Southern Hemisphere, HESS consists of four identical $13 \mathrm{~m}$ diameter Cherenkov telescopes (Bernlöhr et al. 2003). HESS employs the stereoscopic imaging atmospheric Cherenkov technique, and is sensitive to $\gamma$-rays above an energy threshold of $\sim 0.1 \mathrm{TeV}$ (Hinton et al. 2004) for observations at zenith. An angular resolution of 5 to $6^{\prime}$ on an event-byevent basis is achieved, and the large field of view (FoV) with $F W H M \sim 3.5^{\circ}$ (Aharonian et al. 2006b) permits survey coverage in a single pointing. A point source sensitivity of $\sim 1 \% \mathrm{Crab}$ flux $\left(\sim 10^{-13} \mathrm{erg} \mathrm{cm}^{-2} \mathrm{~s}^{-1}\right.$ at $\left.1 \mathrm{TeV}\right)$ is achieved for a $5 \sigma$ detection after $\sim 25 \mathrm{~h}$ observation. Further details concerning HESS can be found in Hinton (2004) and references therein.

HESS observed Coma during the 2006 season for a total of $8.2 \mathrm{~h}$ (corrected for the detector deadtime) comprising 19 runs of duration $\sim 28 \mathrm{~min}$ each. Those runs were accepted for data analysis if they met the quality control criteria described in Aharonian et al. (2004). Data were analysed using the momentbased Hillas analysis procedure described in Aharonian et al. (2006b). Minimum cuts on the Cherenkov image $\operatorname{size}^{1}$ of 80 and 200 photoelectrons corresponding to standard and hard cuts were employed. The average zenith angle of the dataset was $\sim 53^{\circ}$ yielding energy thresholds (peak detection rate for a power law source spectrum with an exponent of 2.1) of $\sim 1.1 \mathrm{TeV}$ and $\sim 2.3 \mathrm{TeV}$ for standard and hard cuts analyses. This analysis follows on from preliminary HESS results (Domainko et al. 2007).

The large FoV of HESS is well-suited to Coma as TeV emission could be expected from a variety of sites - the central radio halo or core; the radio-relic and adjacent galaxy merger/infall region; the degree-scale accretion shock suspected to surround the cluster (e.g. Voit 2005), and individual member galaxies. $\mathrm{TeV} \gamma$-ray significance skymaps covering a $7^{\circ} \times 7^{\circ} \mathrm{FoV}$ (from a mosaic of pointings) are presented in Fig. 1), employing oversampling radii of $0.2^{\circ}$, appropriate for moderately extended sources in the Coma field. Skymaps employing a $0.1^{\circ}$ oversampling radius (Fig. A.1) for pointlike sources are available in the appendix. The CR background estimate in skymaps shown here is based on the template-model (Rowell 2003), employing a region spatially overlapping the source region but not containing any $\gamma$-ray-like events. Also available in the appendix are the distributions of skymap significances (Fig. A.2) which are wellexplained by Gaussians with standard deviation within a few percent of unity and means very close to zero, indicating that the background estimate performs well over the FoV. Similar results were also obtained when employing alternative CR background estimates such as the ring/ring-segment and reflected region models (Berge et al. 2007) which were used for upper limit calculations (in Table 1). Results were also cross-checked using an alternative analysis chain.

Table 1 summarises results for various locations in the Coma field guided by results from the ROSAT all sky survey (Briel et al. 1992; Voges et al. 1999), XMM-Newton observations (Feretti \& Neumann 2007) and Arecibo-DRAO radio observations (Kronberg et al. 2007). The HESS TeV excess significance $S$ and flux upper limits $\Phi^{99 \%}$ (for an $E^{-2.1}$ spectrum and using the method of Feldman \& Cousins 1998) for $E>1,>5$, and

1 Total photoelectron signal in the Cherenkov image. 

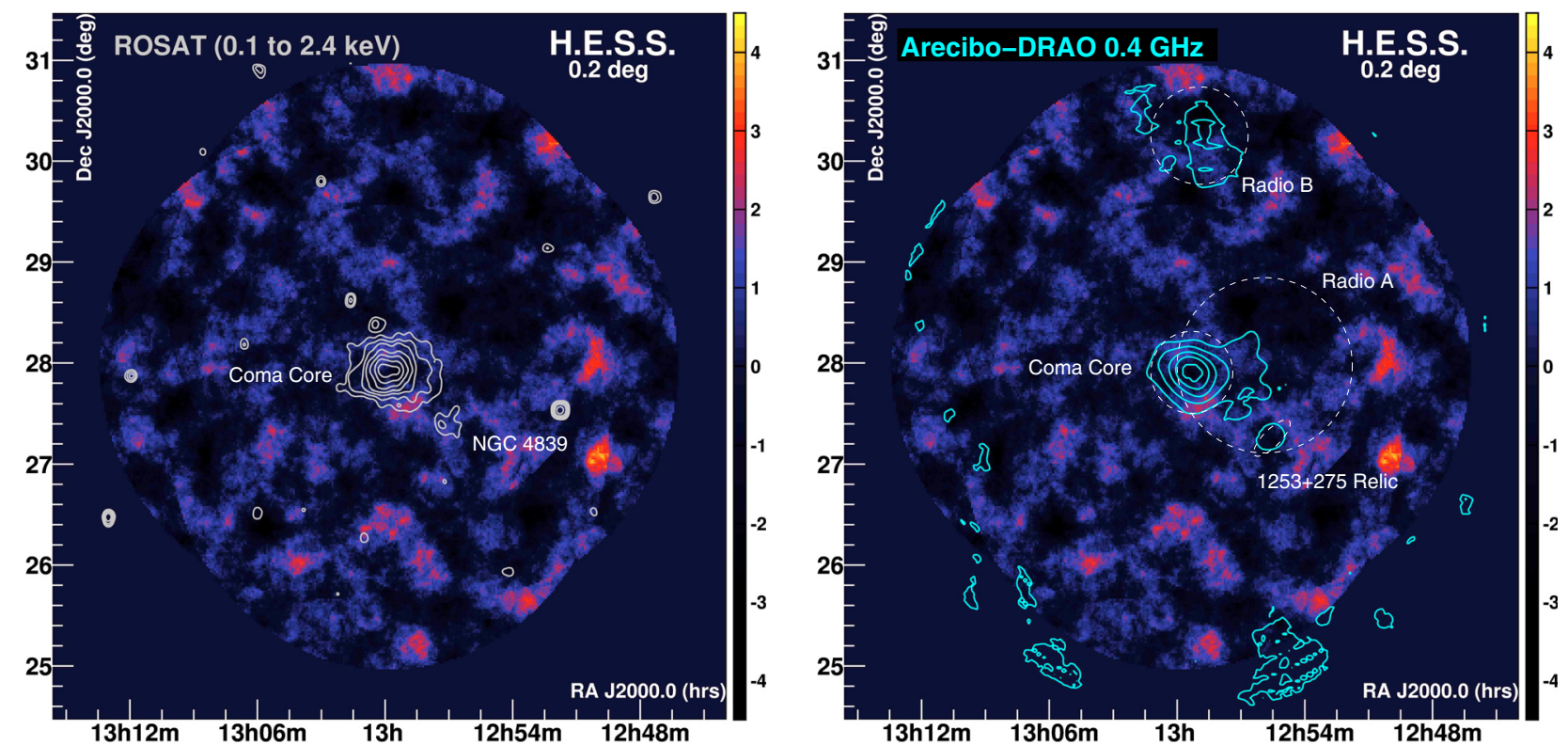

Fig. 1. Left: skymap of HESS TeV excess significance (colour-scale over $\pm 4 \sigma$ ) calculated using Li \& Ma (1983) over a $7^{\circ} \times 7^{\circ}$ FoV, employing the template CR background model (Rowell 2003). An oversampling radius of $0.2^{\circ}$ was used, appropriate for extended source searching. Overlaid contours (light-grey solid lines) represent total band (0.1 to $2.4 \mathrm{keV})$ smoothed X-ray counts $\mathrm{s}^{-1}$ in $\log$-scale from the ROSAT all-sky survey (Voges et al. 1999). Right: as for Left but with overlaid contours from radio observations $(0.4 \mathrm{GHz}-\mathrm{K}$ contours rebinned from the original above $2.9 \mathrm{~K}$ or $\sim 11 \sigma$ ) from Kronberg et al. (2007) with strong point sources removed. The white dashed circle indicates the intrinsic $0.4^{\circ}$ radius source size and position for the Coma Core (Table 1).

Table 1. Numerical summary for various regions in the Coma galaxy cluster and surrounding field.

\begin{tabular}{|c|c|c|c|c|c|c|c|c|c|c|c|}
\hline Name & $1 \mathrm{RXS}$ & $\begin{array}{c}\text { RA } \\
{[\mathrm{J} 2000.0]}\end{array}$ & $\begin{array}{c}\text { Dec } \\
{[\mathrm{J} 2000.0]}\end{array}$ & ${ }^{1}{ }^{1}$ RoI [deg] & ${ }^{2} T[\mathrm{~h}]$ & \multicolumn{3}{|c|}{$\begin{array}{c}{ }^{3} S[\sigma] \\
(E>1,5,10 \mathrm{TeV})\end{array}$} & \multicolumn{3}{|c|}{$\begin{array}{c}\text { Flux U.L. }{ }^{4} \Phi^{99 \%} \\
(E>1,5,10 \mathrm{TeV}) \\
\end{array}$} \\
\hline Coma Core & $\mathrm{J} 125947.9+275636$ & $12^{\mathrm{h}} 59^{\mathrm{m}} 47.9^{\mathrm{s}}$ & $27^{\circ} 56^{\prime} 36^{\prime \prime}$ & 0.0 & 7.3 & -0.5 & -1.2 & -1.4 & 6.1 & 0.3 & 0.1 \\
\hline & & & & 0.2 & 7.3 & +0.4 & -0.4 & -0.6 & 10.8 & 0.9 & 0.5 \\
\hline & & & & 0.4 & 7.3 & +1.1 & -0.5 & -1.5 & 25.5 & 1.7 & 0.6 \\
\hline $1253+275-$ Relic $^{\S}$ & & $12^{\mathrm{h}} 55^{\mathrm{m}} 15.0^{\mathrm{s}}$ & $27^{\circ} 15^{\prime} 00^{\prime \prime}$ & $\S$ & 5.7 & +1.3 & +2.0 & +1.0 & 15.9 & 2.6 & 1.2 \\
\hline Radio-A & & $12^{\mathrm{h}} 55^{\mathrm{m}} 00.0^{\mathrm{s}}$ & $28^{\circ} 00^{\prime} 00^{\prime \prime}$ & 0.9 & 4.6 & +2.0 & -2.2 & -2.0 & 78.7 & 2.3 & 1.4 \\
\hline Radio-B & & $13^{\mathrm{h}} 00^{\mathrm{m}} 00.0^{\mathrm{s}}$ & $30^{\circ} 15^{\prime} 00^{\prime \prime}$ & 0.5 & 1.6 & +2.5 & +0.7 & +0.0 & 77.8 & 7.0 & 3.4 \\
\hline NGC 4839 & $\mathrm{~J} 125710.8+272426$ & $12^{\mathrm{h}} 57^{\mathrm{m}} 24.3^{\mathrm{s}}$ & $27^{\circ} 29^{\prime} 52^{\prime \prime}$ & 0.2 & 6.8 & +0.4 & +1.7 & +1.2 & 9.0 & 1.9 & 1.0 \\
\hline & & & & 0.4 & 6.8 & -1.8 & +0.3 & +0.1 & 6.7 & 2.0 & 1.3 \\
\hline
\end{tabular}

1. Source Region of Interest (RoI) intrinsic radius. Actual radii used are convolved with the analysis PSF. A zero value here refers to a point-source analysis.

2. Observation time (h) corrected to a $0.7^{\circ}$ off-axis angle using a standard cuts $E>1 \mathrm{TeV}$ response curve.

3. Statistical significance using Li \& Ma (1983).

4. $99 \%$ C.L. flux upper limit $\times 10^{-13} \mathrm{ph} \mathrm{cm}^{-2} \mathrm{~s}^{-1}$.

$\S$ Elliptical region $\left(0.33^{\circ} \times 0.2^{\circ}\right.$ with position angle $\left.45^{\circ}\right)$ as defined in Feretti \& Neumann (2007).

$>10 \mathrm{TeV}$ were taken from standard, hard, and hard cuts analyses respectively. CR background estimates were taken from the reflected model (Berge et al. 2007).

In X-rays, extended emission from the Coma cluster and emission further to the southwest are evident. The southwest thermal X-ray emission is not entirely spatially coincident with the radio-relic (discussed below), but is centred on the galaxy sub-group NGC 4839 (labeled NGC 4839 in Table 1), 20' closer to the Coma cluster core compared to the radio-relic. This sub-group is thought to represent a merger or infall of galaxies associated with Coma. Hard X-ray (18-30 keV) observations with INTEGRAL (Eckert et al. 2007) suggest excess emission in the direction of this infalling sub-group close to the cluster centre. In radio, the Coma core is visible as well as a radio extension to the southwest known as the radio relic region (labeled 1253+275-Relic in Table 1). Two additional regions were also chosen to overlap the diffuse radio features labeled " $A$ " and "B" from Kronberg et al. (2007, at $0.4 \mathrm{GHz}$ ) for which circular regions of radii $0.9^{\circ}$ and $0.5^{\circ}$ respectively were used. Radio " $\mathrm{A}$ " appears to well encompass the radio core of Coma which is discussed at length in Thierbach et al. (2003) from their $>2 \mathrm{GHz}$ observations, whilst radio " $\mathrm{B}$ " is a new feature from Kronberg et al. (2007). In all cases no evidence for $\mathrm{TeV}$ emission was seen and 99\% confidence level flux upper limits (assuming an $E^{-2.1}$ spectrum) at several energy thresholds $(E>1,>5$ and $>10 \mathrm{TeV}$ ) were set. We note the highest excess significance feature at $\sim 4.1 \sigma$ towards $\mathrm{RA}=12^{\mathrm{h}} 55^{\mathrm{m}}$ Dec $=+27^{\circ} 15^{\prime}$ is expected by chance given the number of independent trials $\left(\sim 10^{5}\right)$ in the image.

\section{Discussion}

One of the most important properties of clusters of galaxies is the fact that $\mathrm{CR}$ protons remain diffusively confined in the magnetised intracluster medium for cosmological time scales. The maximum energy that can be confined depends on the 
Table 2. Constraints ${ }^{\dagger}$ on the ratio of CR (non-thermal) to thermal energy $\left(E_{\mathrm{CR}} / E_{\mathrm{th}}\right)$ for the Coma cluster core region (within two radii) and assumed cosmic-ray distribution models A and B (see text).

\begin{tabular}{ccccc}
\hline \hline Radius & $\alpha$ & Model & $\eta=E_{\mathrm{CR}} / E_{\text {th }}$ & $E_{\mathrm{CR}}[\mathrm{erg}]$ \\
\hline $0.2^{\circ}(0.33 \mathrm{Mpc})$ & 2.1 & $\mathrm{~A}$ & $<0.19$ & $<7.4 \times 10^{61}$ \\
$0.4^{\circ}(0.67 \mathrm{Mpc})$ & 2.1 & $\mathrm{~A}$ & $<0.18$ & $<2.5 \times 10^{62}$ \\
$0.4^{\circ}(0.67 \mathrm{Mpc})$ & 2.1 & $\mathrm{~B}$ & $<0.25$ & $<3.5 \times 10^{62}$ \\
$0.4^{\circ}(0.67 \mathrm{Mpc})$ & 2.3 & $\mathrm{~A}$ & $<0.55$ & $<7.7 \times 10^{62}$ \\
\hline
\end{tabular}

$\dagger$ The upper limit for $E>5 \mathrm{TeV}$ has been used here.

(unknown) diffusion coefficient but an often made assumption is that the maximum energy is well above that relevant for TeV $\gamma$-ray emission (Völk et al. 1996). CR protons lose their energy mainly via proton-proton interactions in the intergalactic medium. Due to the low density of this medium, the energy loss time is longer than the Hubble time. This implies that the hadronic CR content of a cluster is simply the superposition of the contributions from all the CR sources which have been active during the cluster lifetime, with little attenuation due to energy losses. Under reasonable assumptions on the CR acceleration efficiency, the total non-thermal energy stored in the intracluster medium might be of the same order of magnitude of the thermal energy. For example Ryu et al. (2003) have estimated a non-thermal energy fraction reaching $50 \%$ of the thermal energy. Such an amount of CR protons would result in copious emission of $\gamma$-rays from the decay of neutral pions produced in proton-proton interactions. Since the most optimistic theoretical predictions are well within the capabilities of current-generation Cherenkov telescopes, the upper limits obtained by HESS can be used effectively to constrain the non-thermal energy content of the Coma cluster.

A remarkable feature of the $\gamma$-ray emission from neutral pion decay is that its spatial profile is expected to follow the density profile of the gas which constitutes the target for proton-proton interactions. In the case of the Coma cluster, this gas is concentrated within a core of radius $\sim 300 \mathrm{kpc}$ which, at the distance of the Coma cluster, corresponds to $\sim 0.2^{\circ}$ (see discussion below). This is the basis for the angular regions from which upper limits have been extracted.

Table 2 demonstrates how the upper limits on the $\gamma$-ray emission convert into upper limits on the ratio of the cluster thermal energy to that of CR protons (non-thermal energy). This non-thermal to thermal energy ratio is denoted $\eta=E_{\mathrm{CR}} / E_{\mathrm{th}}$. The Coma cluster thermal energy has been evaluated using the gas density profile and the intracluster medium temperature derived from X-ray data (e.g. Neumann et al. 2003) and resulted in $E_{\text {th }} \sim 3.9 \times 10^{62}$ and $1.4 \times 10^{63}$ erg for regions within 0.2 and 0.4 degrees from the cluster centre respectively. The expected $\gamma$-ray emission has been computed following Kelner et al. (2006) and assuming that the energy spectrum for CR protons is a single power law with spectral index $\alpha=2.1$ and 2.3 starting at an energy of $1 \mathrm{GeV}$. The assumption of such hard spectra is justified by the fact that, due to $\mathrm{CR}$ confinement within the intracluster medium, the equilibrium spectrum must be equal to the CR injection spectrum at the sources. Note that much steeper spectra ( $\alpha$ up to 6) are indicated for CRs accelerated at weak merger shocks (Gabici \& Blasi 2003b; Berrington \& Dermer 2003). The cluster non-thermal energy has been obtained by integrating the spectrum above $1 \mathrm{GeV}$ and the resulting $\gamma$-ray emission corrected for absorption in the cosmic infrared background (CIB) using the Salpeter initial mass function opacity given in
Primack (2001). More recent constraints on the CIB from the TeV blazar 1ES 0229+200 (Aharonian et al. 2007) provide only a negligible change in absorption from 1 to $10 \mathrm{TeV}$ given Coma's proximity. Our upper limits can then be used to constrain this overall CR spectrum and hence non-thermal energy.

The only missing piece of information is the spatial distribution of CRs. This quantity is not unambiguously known and it depends on the spatial distribution of CR sources in clusters. Here, two distinct situations were considered as two extreme cases. In the first one, referred to as Model-A, the radial profile of the CR energy density was assumed to follow the thermal energy profile. In Model-B, a spatially homogeneous distribution for the CRs was assumed.

In all cases considered, the most constraining data points in terms of energy flux are the upper limits for photon energies above $5 \mathrm{TeV}$. Our $>10 \mathrm{TeV}$ limits are marginally higher $(\sim 20 \%)$ whilst the $>1 \mathrm{TeV}$ limits are factor 2 to 3 higher. For a CR spectrum with $\alpha=2.1$ and assuming that the CR energy density follows the thermal energy density (Model-A) values for $\eta \lesssim 0.2$ for both the considered regions $\left(0.2^{\circ}\right.$ and $\left.0.4^{\circ}\right)$ were obtained. In order to check how the upper limits depend on the assumptions made on the CR spectrum and spatial distribution, the size of the region was fixed to $0.4^{\circ}$ and two more cases were considered: a homogeneous distribution of CRs (Model-B) and a softer CR spectrum with $\alpha=2.3$. The upper limits on $\eta$ are in these cases less stringent and are reported in the third and fourth rows of Table 2 , resulting in $\eta \lesssim 0.25$ and 0.55 respectively. In combination with our limits for $E>1 \mathrm{TeV}$ and $>10 \mathrm{TeV}$, modelindependent constraints on the $\gtrsim 10, \gtrsim 50, \gtrsim 100 \mathrm{TeV}$ CR proton population in Coma were set.

Our upper limits can be compared with those obtained from observations at other wavelengths and also other models. Firstly, our limits are slightly more constraining compared to those obtained from EGRET MeV/GeV data $(\eta=0.45$ and 0.25 assuming $\alpha=2.1,2.3$; Pfrommer \& Enßlin 2004). Our limits also rule out some of the models for CR acceleration in clusters of galaxies that predict high $\eta$ values, even up to $50 \%$ (Ryu et al. 2003). The high frequency non-thermal radio emission of the Coma cluster (Thierbach et al. 2003) has been used by Reimer et al. (2004) to constrain $\eta$ by noticing that the radio emission from secondary electrons produced by CRs in proton-proton interactions cannot exceed the measured value. Recent observations (Brunetti et al. 2008) of a steep radio spectrum from the cluster Abell 521 have also been considered in a similar way as in the Reimer et al. work on Coma. Their rather stringent limits obtained using radio data ( $\eta=10^{-4}$ to $\sim 0.3$ ) depend quadratically on the value of the intracluster $B$-field which has a large uncertainty of a factor $\sim 10(B=0.1$ to $2 \mu \mathrm{G})$. Additionally the $<10 \mathrm{GHz}$ radio measurement constrains formally the $<0.1 \mathrm{TeV}$ $\mathrm{CR}$ population within the range of $B$-fields used. ULs from HESS and other VHE gamma-ray instruments make direct constraints on the $E \gtrsim 10 \mathrm{TeV}$ CR population, energies well above that implied by Reimer et al. (2004), and are essentially independent of the $B$-field. Note that an additional preliminary $E>0.3 \mathrm{TeV}$ upper limit from $\sim 19 \mathrm{~h}$ of VERITAS (Perkins et al. 2008) observations for the Coma core $\left(0.3^{\circ}\right.$ radius $)$ has been reported at $\sim 3 \% \mathrm{Crab}$ (or $\sim 2 \times 10^{-12} \mathrm{ph} \mathrm{cm}^{-2} \mathrm{~s}^{-1}$ ). This VERITAS limit, when converted to an energy flux, provides a constraint on $\eta$ very similar to ours, albeit for CRs of slightly lower energies $\gtrsim$ few TeV. Finally, our $\eta$ constraints for Coma are within a factor of two to three larger than those obtained from somewhat deeper HESS observations $(\sim 33 \mathrm{~h})$ on the Abell 85 cluster $\left(M=7.6 \times 10^{14} M_{\odot} ; z=0.055\right)$ (Aharonian et al. 2009). In this work, the constraints $\eta<0.06$ to $\eta<0.15$ for Abell 85 were 
reported for a range of mass profiles and a CR spectral index of -2.1 .

\section{Conclusions}

HESS observed the Coma galaxy cluster for $\sim 8 \mathrm{~h}$, obtaining upper limits to the $E>1,>5$, and $>10 \mathrm{TeV} \gamma$-ray flux from the cluster core $\left(0.2^{\circ}\right.$ and $0.4^{\circ}$ radii). Additional regions for which upper limits are given include the radio-relic $(1253+275)$; the merger/infall region associated with NGC 4839; and two large-scale radio features "radio-A" $\left(0.9^{\circ}\right.$ radius $)$ and "radio-B" ( $0.5^{\circ}$ radius $)$.

Our results were compared to a model for the proton-proton $\gamma$-ray emission assuming a proton spatial profile matching the centrally peaked thermal gas and injection spectral index of 2.1. In this case our $E>5 \mathrm{TeV}$ HESS upper limit for the Coma core region within a $0.2^{\circ}$ radius region (amounting to $\sim 8 \% \mathrm{Crab}$ flux units or $\sim 10^{-13} \mathrm{ph} \mathrm{cm}^{-2} \mathrm{~s}^{-1}$ ) constrains the fraction of energy $\eta$ in CRs to $<0.2$ times the thermal intracluster medium energy, or $\sim 10^{62} \mathrm{erg}$. This can be compared with the generally more stringent constraints on $\eta$ so far from radio observations (Reimer et al. 2004) in the range $\eta=10^{-4}$ to $\sim 0.3$ times the thermal energy for $B$-fields 0.1 to $2 \mu \mathrm{G}$ in the intracluster medium and proton injection spectral index $\alpha=2.1$ to 2.5 . It should be noted that the HESS $E>5 \mathrm{TeV}$ upper limit assuming $\alpha=2.1$ formally constrains $\gtrsim 50 \mathrm{TeV}$ CRs whilst the radio limits pertain to $<0.1 \mathrm{TeV}$ CRs, highlighting the complementarity of the two approaches. The HESS constraints for the amount of CRs stored in the intracluster medium also rule out the most optimistic theoretical models for CR acceleration in clusters of galaxies.

Our upper limit for the NGC 4839 merger/infall group may also be useful, in conjunction with the hard X-ray emission, in constraining models for the additional shock acceleration of particles potentially associated with this region.

Our focus here has been on the expected centrally peaked proton-proton $\gamma$-ray emission, but emission expected from the inverse-Compton scattering of electrons, and UHE proton $/ \gamma$ interactions $\mathrm{p} \gamma \rightarrow \mathrm{e}^{+} \mathrm{e}^{-}+\mathrm{p}^{\prime}$ may follow the spatial profile of the degree-scale cluster accretion shock as an annulus of radius $\sim 1-2^{\circ}$. Inhomogeneities in the shock structure leading to $\mathrm{TeV}$ hotspots of up to degree in size (Keshet et al. 2003), and uncertainties in the shock size prevented a specific attempt to search for such emission. TeV $\gamma$-ray observations can in principle provide direct constraints on the ability of such shocks to accelerate particles to multi- $\mathrm{TeV}$ energies and beyond. The significance skymap presented in Fig. 1 for $0.2^{\circ}$ radii sources would suggest however that no such evidence for moderate-scale emission in the Coma field is present. Indeed, similar skymaps are obtained for a $0.4^{\circ}$ radius.

Overall our results indicate that deeper observations (towards $100 \mathrm{~h}$ ) of the Coma cluster, or other similarly massive and nearby galaxy cluster in $\mathrm{TeV} \gamma$-rays, are warranted to probe the Universe's largest-scale shocks. Such a detection appears possible unless the total energy in the form of multi-TeV CRs is significantly less than $\sim 10 \%$ of the cluster thermal energy.

Finally, the recently launched LAT instrument onboard the Fermi GST will provide critical constraints in the $\mathrm{MeV} / \mathrm{GeV}$ band (likely within its first year or so of observation) and these results are eagerly awaited.

Acknowledgements. The support of the Namibian authorities and of the University of Namibia in facilitating the construction and operation of HESS is gratefully acknowledged, as is the support by the German Ministry for Education and Research (BMBF), the Max Planck Society, the French Ministry for Research, the CNRS-IN2P3 and the Astroparticle Interdisciplinary Programme


Fig. A.1. HESS signficance skymaps as for Fig. 1 but using an oversampling radius of $0.1^{\circ}$. Top: overlaid contours (light-grey solid lines) represent total band ( 0.1 to $2.4 \mathrm{keV}$ ) smoothed X-ray counts $\mathrm{s}^{-1}$ in logscale from the ROSAT all-sky survey (Voges et al. 1999). Bottom: overlaid contours from radio observations from Kronberg et al. (2007) with strong point sources removed.

of the CNRS, the UK Particle Physics and Astronomy Research Council (PPARC), the IPNP of the Charles University, the Polish Ministry of Science and Higher Education, the South African Department of Science and Technology and National Research Foundation, and by the University of Namibia. We appreciate the excellent work of the technical support staff in Berlin, Durham, Hamburg, Heidelberg, Palaiseau, Paris, Saclay, and in Namibia in the construction and operation of the equipment. We thank Philipp Kronberg for the $408 \mathrm{MHz}$ radio image.

\section{Appendix A: Additional figures}

HESS images as for Fig. 1 but using a $0.1^{\circ}$ integration radius are presented here as well as distributions of significances for both integration radii. 




Fig. A.2. Distributions of HESS significance from Figs. 1 and A.1 for various oversampling radii - top: $0.2^{\circ}$; bottom: $0.1^{\circ}$. The dashed line represents a Gaussian of zero mean and unit standard deviation.

\section{References}

Aharonian, F. 2002, MNRAS, 332, 215

Aharonian, F., Akhperjanian, A. G., Aye, K.-M., et al. (HESS Collab.) 2004, Astropart. Phys., 22, 109

Aharonian, F., Akhperjanian, A. G., Bazer-Bachi, A. R., et al. (HESS Collab.) 2006b, A\&A, 457, 899

Aharonian, F., Akhperjanian, A. G., Barres de Almeida, U., et al. (HESS Collab.) 2007, 475, L9

Aharonian, F., et al. (HESS Collab.) 2009, A\&A, 495, 27
Ajello, M., Rebusco, P., Cappelluti, N., et al. 2009, ApJ, 690, 367 Atoyan, A. M., \& Völk, H. J. 2000, ApJ, 535, 45

Berezinsky, V. S., Blasi, P., \& Ptuskin, V. S. 1997, ApJ, 487, 529

Berge, D., Funk, S., \& Hinton, J. 2007, A\&A, 466, 1219

Bernlöhr, K., et al. 2003, APh, 20, 111

Berrington, R., \& Dermer, C. D. 2003, ApJ, 594, 709

Blasi, P. 1999, ApJ, 525, 603

Blasi, P., Gabici, S., \& Brunetti, G. 2007, Int. J. Mod. Phys., A22, 681

Briel, U. G., Henry, J. P., \& Böhringer, H. 1992, A\&A, 259, L31

Brunetti, G., \& Blasi, P. 2005, MNRAS, 363, 117

Brunetti, G., Giacintucci, S., Cassano, R., et al. 2008, Nature, 455, 944

Colafrancesco, S., \& Blasi, P. 1998, APh, 9, 227

Colafrancesco, S., Profumo, S., \& Ullio, P. 2006, A\&A, 455, 21

Dennison, B. 1980, ApJ, 239, L93

Domainko, W., et al. 2007, in Proc. 30th ICRC (Mexico) [arXiv : 0708: 1384]

Eckert, D., Neronov, A., Courvoiier, T. L.-J., \& Produit, N. 2007, A\&A, 470, 835

Enßlin, T. A., Biermann, P. L., Kronberg, P. P., \& Wu, X. P. 1997, ApJ, 477, 560

Feldman, G. J., \& Cousins, R. D. 1998, Phys. Rev. D, 57, 3873

Feretti, L., \& Neumann, D. M. 2006, A\&A, 450, L21

Feretti, L., Brunetti, G., Giovannini, G., et al. 2004, JKAS, 37, 315

Fusco-Femiano, R., Orlandini, M., \& Brunetti, G. 2004, ApJ, 602, 73

Gabici, S., \& Blasi, P. 2003a, APh, 19, 679

Gabici, S., \& Blasi, P. 2003b, ApJ, 583, 695

Gabici, S., \& Blasi, P. 2004, APh, 20, 579

Giovannini, G., Feretti, L., Venturi, T., et al. 1993, ApJ, 406, 399

Hillas, A. M. 1984, ARA\&A, 22, 425

Hinton, J. A. 2004, New Astron. Rev., 48, 331

Hinton, J. A., Domainko, W., \& Pope, E. C. 2007, MNRAS, 382, 466

Inoue, S., Aharonian, F., \& Sugiyama, N. 2005, ApJ, 628, L9

Kang, H., Ryu, D., \& Jones, T. W. 1996, ApJ, 456, 422

Kelner, S. R., \& Aharonian, F. A. 2008, Phys. Rev. D, 78, 034013

Kelner, S. R., Aharonian, F. A., \& Bugayov, V. V. 2006, Phys. Rev. D, 74, 034018

Keshet, U., Waxman, E., Loeb, V., et al. 2003, ApJ, 585, 128

Kronberg, P. P., Kothes, R., Salter, C. J., \& Perillat, P. 2007, ApJ, 659, 267

Loeb, A., \& Waxman, E. 2000, Nature, 405, 156

Li, T., \& Ma, Y. 1983, ApJ, 272, 317

Lutovinov, A. A., et al. 2008 [arXiv:0802 . 3742]

Neumann, D. A., Lumb, D. H., Pratt, G. W., \& Briel, U. G. 2003, A\&A, 400, 811

Perkins, J., Badran, H., Blaylock, G., et al. 2006, ApJ, 644, 148

Perkins, J., et al. (VERITAS Coll.) 2008, in Proc. Heidelberg Gamma-Ray Symp. [arXiv: 0810.0302]

Pfrommer, C., \& Enßlin, T. A. 2004, MNRAS, 352, 76

Primack, J. 2001, AIP Conf., 558, 463

Reimer, O., Pohl, M., Sreekumar, P., \& Mattox, J. R. 2003, ApJ, 588, 155

Reimer, A., Reimer, O., Schlickeiser, R., \& Iyudin, A. 2004, A\&A, 424, 773

Rephaeli, M., \& Gruber, D. 2002, ApJ, 597, 587

Rossetti, Y., \& Molendi, S. 2004, A\&A, 414, L41

Rowell, G. P. 2003, A\&A, 410, 389

Ryu, D., Kang, H., Hallman, E., \& Jones, T. W. 2003, ApJ, 593, 599

Thierbach, M., Klein, U., \& Wielebinski, R. 2003, A\&A, 397, 53

Völk, H., Aharonian, F. A., \& Breitschwerdt, D. 1996, SSRv, 75, 279

Voges, W., Aschenbach, B., Boller, Th., et al. 1999, A\&A, 349, 389

Voit, G. M. 2005, Rev. Mod. Phys., 77, 207

1 Max-Planck-Institut für Kernphysik, PO Box 103980, 69029 Heidelberg, Germany

2 Dublin Institute for Advanced Studies, 5 Merrion Square, Dublin 2, Ireland

3 Yerevan Physics Institute, 2 Alikhanian Brothers St., 375036 Yerevan, Armenia

4 Universität Erlangen-Nürnberg, Physikalisches Institut, ErwinRommel-Str. 1, 91058 Erlangen, Germany

5 University of Durham, Department of Physics, South Road, Durham DH1 3LE, UK

6 Centre d'Étude Spatiale des Rayonnements, CNRS/UPS, 9 Av. du Colonel Roche, BP 4346, 31029 Toulouse Cedex 4, France

7 Astroparticule et Cosmologie (APC), CNRS, Université Paris 7 Denis Diderot, 10, rue Alice Domon et Leonie Duquet, 75205 Paris Cedex 13; UMR 7164 (CNRS, Université Paris VII, CEA, Observatoire de Paris), France

8 Landessternwarte, Universität Heidelberg, Königstuhl, 69117 Heidelberg, Germany 
9 Institut für Physik, Humboldt-Universität zu Berlin, Newtonstr. 15, 12489 Berlin, Germany

10 LUTH, Observatoire de Paris, CNRS, Université Paris Diderot, 5 Place Jules Janssen, 92190 Meudon, France

11 IRFU/DSM/CEA, CE Saclay, 91191 Gif-sur-Yvette, Cedex, France

12 Nicolaus Copernicus Astronomical Center, ul. Bartycka 18, 00-716 Warsaw, Poland

13 Unit for Space Physics, North-West University, Potchefstroom 2520, South Africa

14 Laboratoire d'Astrophysique de Grenoble, INSU/CNRS, Université Joseph Fourier, BP 53, 38041 Grenoble Cedex 9, France

15 LPNHE, Université Pierre et Marie Curie Paris 6, Université Denis Diderot Paris 7, CNRS/IN2P3, 4 Place Jussieu, 75252 Paris Cedex 5, France

16 Laboratoire Leprince-Ringuet, École Polytechnique, CNRS/IN2P3, 91128 Palaiseau, France

17 Laboratoire d'Annecy-le-Vieux de Physique des Particules, CNRS/IN2P3, 9 Chemin de Bellevue, BP 110, 74941 Annecy-le-Vieux Cedex, France

18 University of Namibia, Private Bag 13301, Windhoek, Namibia

19 Instytut Fizyki Ja̧drowej PAN, ul. Radzikowskiego 152, 31-342 Kraków, Poland

20 Laboratoire de Physique Théorique et Astroparticules, Université Montpellier 2, CNRS/IN2P3, CC 70, Place Eugène Bataillon, 34095 Montpellier Cedex 5, France
21 Universität Hamburg, Institut für Experimentalphysik, Luruper Chaussee 149, 22761 Hamburg, Germany

22 School of Physics \& Astronomy, University of Leeds, Leeds LS2 9JT, UK

23 Institut für Astronomie und Astrophysik, Universität Tübingen, Sand 1, 72076 Tübingen, Germany

24 Department of Physics, Kyoto University, Oiwake-cho, Kitashirakawa, Sakyo-ku, Kyoto 606-8502, Japan

25 Torun Centre for Astronomy, Nicolaus Copernicus University, ul. Gagarina 11, 87-100 Toruń, Poland

26 Charles University, Faculty of Mathematics and Physics, Institute of Particle and Nuclear Physics, V Holešovičkách 2, 18000 Prague, Czech Republic

27 European Associated Laboratory for Gamma-Ray Astronomy, jointly supported by CNRS and MPG

28 Obserwatorium Astronomiczne, Uniwersytet Jagielloński, ul. Orla 171, 30-244 Kraków, Poland

29 Stanford University, HEPL and KIPAC, Stanford, CA 94305-4085, USA

30 School of Chemistry \& Physics, University of Adelaide, Adelaide 5005, Australia e-mail: growell@physics.adelaide.edu.au

31 Institut für Theoretische Physik, Lehrstuhl IV: Weltraum und Astrophysik, Ruhr-Universität Bochum, 44780 Bochum, Germany 